EPSC Abstracts

Vol. 15, EPSC2021-609, 2021, updated on 11 Jan 2022

https://doi.org/10.5194/epsc2021-609

Europlanet Science Congress 2021

(C) Author(s) 2022. This work is distributed under

the Creative Commons Attribution 4.0 License.

\title{
The stellar occultations by the largest satellite of the dwarf planet Haumea, Hi'iaka
}

Estela Fernández-Valenzuela ${ }^{1}$, Jose Luis Ortiz $^{2}$, Bryan Holler ${ }^{3}$, Monica Vara-Lubiano ${ }^{2}$, Nicolas Morales ${ }^{2}$, Bruno Sicardy ${ }^{4}$, Frederic Vachier ${ }^{5}$, Josselin Desmars ${ }^{5,6}$, Felipe Braga-Ribas ${ }^{4,7}$, Zafar Rustamkulov ${ }^{8}$, Alex de la Vega ${ }^{9}$, Elizabeth Warner ${ }^{10}$, Dennis Conti ${ }^{11}$, Jean Lecacheux ${ }^{4}$, Richard Francini ${ }^{12}$, Phil Langill ${ }^{13}$, Ruben Morales ${ }^{14}$, Emmanuel Jehin ${ }^{15}$, Wesley C. Fraser ${ }^{16}$, Damya Souami ${ }^{4}$, and the Lucky Star and Hi'iaka's occultation Teams ${ }^{*}$

${ }^{1}$ University of Central Florida, Florida Space Institute, Orlando, United States of America (estela@ucf.edu)

${ }^{2}$ Instituto de Astrofísica de Andalucía, Consejo Superior de Investigaciones Científicas, Granada, Spain

${ }^{3}$ Space Telescope Science Institute, Baltimore, MD, USA

${ }^{4}$ LESIA, Observatoire de Paris, PSL Research University, CNRS, Sorbonne Université, Univ. Paris Diderot, Sorbonne Paris Cité, France

${ }^{5}$ Institut de mécanique céleste et de calcul des éphémérides, University of Paris, Paris, France

${ }^{6}$ Institut Polytechnique des Sciences Avancées IPSA, 63 boulevard de Brandebourg, F-94200 Ivry-sur-Seine, France.

${ }^{7}$ Observatório Nacional/MCTIC, Rio de Janeiro - RJ, Brazil

${ }^{8}$ Department of Earth and Planetary Science, Johns Hopkins University, Baltimore, MD, USA

${ }^{9}$ Department of Physics and Astronomy, Johns Hopkins University, Baltimore, MD, USA

${ }^{10}$ The University of Maryland, College Park, MD, USA

${ }^{11}$ American Association of Variable Star Observers (AAVSO)

${ }^{12}$ Astronomical League and American Association of Variable Star Observers (AAVSO)

${ }^{13}$ Rothney Astrophysical Observatory, University of Calgary, Alberta, Canada

${ }^{14}$ University of Calgary, Calgary, Alberta, Canada

${ }^{15}$ University of Liege, Liege, Belgium

${ }^{16}$ Herzberg Astronomy and Astrophysics Research Centre, National Research Council of Canada

${ }^{*}$ A full list of authors appears at the end of the abstract

Two stellar occultations by the largest satellite of the dwarf planet Haumea, Hi'iaka, were predicted to happen on April, 6th and 16th, 2021. Additional high accuracy astrometric analysis was carried out in order to refine the prediction for April 6th, using several telescopes in the 1.2-m to 2-m range, with the final shadow path crossing North Africa. We successfully detected the first event from TRAPPIST-North telescope at Oukaïmeden Observatory (Morocco). Although it was recorded from only one site, this first detection allowed us to improve the prediction for the second that crossed North America from East to West. We had a good success recording six positive detections and several negative detections that constrain the shape and size of the body. The light curves obtained from the different observatories provide the time at which the star disappears and reappears, which are translated into chords (the projected lines on the sky-plane as observed from each location). Additionally, we carried out a campaign to study Hi'iaka's rotational light-curve, studying the residuals of Haumea's rotational light-curve to a four-order Fourier fit. We obtained the rotational phases at the times of the occultations, which is critical for the analysis of the occultations, given that Hi'iaka is clearly non-spherical. Our preliminary results show that Hi'iaka 
indeed has a triaxial shape with a larger effective diameter than what has been published so far. The preliminary results and their implications will be discussed in this talk.

Lucky Star and Hi'iaka's occultation Teams: Lucky Star Team members: Pablo Santos-Sanz, René Duffard, Alvaro Alvarez-Candal, Mike Kretlow (Instituto de Astrofísica de Andalucía, Consejo Superior de Investigaciones Científicas, Granada, Spain); Gustavo Benedetti Rossi, Bruno Morgado, Julio Camargo, Flavia L. Rommel, Altair Ramos Gomes Junior (Observatório Nacional/MCTIC, Rio de Janeiro - RJ, Brazil); Marcelo Assafin (Universidade federal do Rio de Janeiro, Observatório do Valongo). Hi'iaka's occultation Team: Djounai Baba Aissa, Zaki Grigahcene (Center of Research in Astronomy, Astrophysics and Geophysics, CRAAG - Algiers Observatory, Algeria); Marc Buie (Southwest Research Institute, San Antonio, TX, USA); Javier Licandro, Miguel Rodriguez-Alarcon, Miquel Serra (Instituto de Astrofísica de Canarias); Alberto Castro-Tirado, Emilio Jesús FernandezGarcia (Instituto de Astrofísica de Andalucía, IAA-CSIC, Spain); Ramón Iglesias-Marzoa, Francisco Galindo (Observatorio de Javalambre OAJ, Teruel, Spain); Luis Pérez (Allariz, Spain); Hugo González (Observatorio de Forcarei, Spain); Pablo Canedo, Óscar Blanco (A Veiga, Spain); Rui Gonçalves (Constancia, Portugal); Adam W Rengstorf (Purdue University Northwest, Hammond, IN, USA); Randy Flynn (Squirrel Valley Observatory, Columbus, North Carolina, USA); Aart M. Olsen, Bill Hanna (IOTA, USA); Jason Barnes, Joseph A. A'Hearn, Steven M. Kreyche, William J. Miller, Lillian E. Mortensen, Thomas C. Gibson (Department of Physics, University of Idaho, Moscow, ID, USA); Gary Walker (Maria Mitchell Observatory in Nantucket, MA, USA); Gregory A. Feiden, Joseph Froetschel, Suzanne Steel, Destin Encardes (University of North Georgia, Dahlonega, GA, USA); Scott Fisher, Alton Luken, Eric Holcomb (Pine Mountain Observatory, Univ. of Oregon, Dept. of Physics, Eugene, OR, USA); Daniel B. Caton (Appalachian State University, Boone, NC, USA); and Bob Dunford. 